\title{
Benzodiazepine Misuse: An Epidemic Within a Pandemic
}

\author{
Ashish Sarangi ${ }^{1}$, Terry McMahon ${ }^{1}$, Jayasudha Gude ${ }^{2}$ \\ 1. Psychiatry, Texas Tech University Health Sciences Center, Lubbock, USA 2. Psychiatry, Zuckerside Hillside Hospital, \\ New York, USA
}

Corresponding author: Ashish Sarangi, aks_sarangi@hotmail.com

\begin{abstract}
Coronavirus disease 2019 (COVID-19) had deleterious effects on patients with mental health problems and several studies have shown a spike in the rates of depression, insomnia, and post-traumatic stress disorder. Anxiety and insomnia rates have also increased among both the general public and health care professionals. Benzodiazepines are some of the most commonly used drugs in the treatment of anxiety and insomnia. However, benzodiazepines are also misused, abused alone, or abused in combination with other drugs. Lockdowns and social distancing have also had negative consequences on patients with mental health problems. We assessed the extent of benzodiazepine use during the pandemic and interpreted its effects in the future. We conducted a literature search using the Preferred Reporting Items for Systematic Reviews and Meta-Analyses (PRISMA) protocol and eight articles reviewed specifically reported worrying fluctuations in benzodiazepine use during the pandemic. We observed varied trends in the usage of benzodiazepines in various parts of the world. Some studies showed an increase in the consumption of benzodiazepine while others demonstrated a decrease in the prescription refills of benzodiazepine, which may be a result of gaps in mental health care. At this time, we can conclude that the current trend with benzodiazepine use is fluctuating and mental health professionals must continue to exercise caution before prescribing benzodiazepines. Future research is also warranted to be aware of the changing patterns and to avoid misuse and/or abuse at an epidemic level.
\end{abstract}

Review began 05/28/2021 Review ended 06/21/2021 Published 06/21/2021

\section{(๑) Copyright 2021}

Sarangi et al. This is an open access article distributed under the terms of the Creative Commons Attribution License CC-BY 4.0., which permits unrestricted use, distribution, and reproduction in any medium, provided the original author and source are credited.
Categories: Psychiatry, Infectious Disease, Public Health

Keywords: benzodiazepine, covid-19, pandemic, coronavirus, psychiatry

\section{Introduction And Background}

It has been more than a year since the coronavirus disease 2019 (COVID-19) was first reported in December of 2019 [1]. According to World Health Organization, there have been 170 million cases reported and almost every country has been affected by the coronavirus pandemic [1,2]. The COVID-19 outbreak has led people everywhere to implement drastic changes in lifestyle, including social distancing. Periods of social isolation and loneliness have resulted in negative consequences on mental well-being. It was found that people were three times more likely to have anxiety or depressive disorders in 2020 compared to the previous year [3], and more than one in three individuals presented one or both disorders. According to the CDC, from January 20,2021 , to February 1,2021 , more than two in five adults aged $\geqslant 18$ years experienced symptoms of anxiety or a depressive disorder during the past seven days [4]. It has also been reported that symptom rates of generalized anxiety, psychological distress, and COVID-19 related fear are $44.9 \%, 65.2 \%$, and $59 \%$ respectively [5]. Since the pandemic onset, there has been also a $37 \%$ increase in the rate of clinical insomnia (from $14.6 \%$ to $20 \%$ ) [6]. Among health care workers caring for COVID-19 patients, there has been an increase in the prevalence of anxiety to $25.8 \%$ (95\% CI $20.5-31.9 \%$ ) and stress to $45 \%$ (95\% CI 24.3 67.5\%) [7].

Benzodiazepines are a class of medications used to treat conditions such as anxiety and insomnia. In 1955, the first benzodiazepine (BZD), chlordiazepoxide, was developed, followed by diazepam in 1963 [8-11]. In 1957, as an alternative to previous barbiturates, BZD use started to gradually replace the opiate derivates [12]. Benzodiazepines became widely used drugs because of their potential benefits and were the most abused drugs by the 1970s [13]. With growing concern about the abuse of BZDs, they were placed on Food and Drug Administration drug list [14]. The risk of BZD dependence was officially recognized by the American Psychiatric Association in 1990 [15,16]. BZDs act by binding to the gamma-aminobutyric acid A (GABA-A) [17] receptors in the brain, inhibiting the brainstem arousal pathways. When BZDs interact with GABA-A receptors, ion channels open more frequently, increasing the inflow of chloride ions which increases membrane polarization and inhibits neuron firing resulting in central nervous system (CNS) depression [15]. This mechanism results in anxiolytic, sedative, hypnotic, anticonvulsant and skeletal muscle-relaxing effects.

Benzodiazepines are classified based on their elimination half-life. They include long-acting diazepam, chlordiazepoxide, flurazepam, and clorazepate along with intermediate-acting alprazolam, clonazepam, lorazepam, oxazepam, and temazepam with short-acting agents being midazolam and triazolam. BZDs are 
metabolized oxidatively in the liver by the cytochrome P450 enzymes (phase I), conjugated with glucuronide (phase II), and excreted almost entirely in the urine [18]. They are prescribed for a wide range of conditions, which include insomnia, agitation, anxiety and convulsions [19]. The dose-related side effects include amnesia and central respiratory depression [18]. Benzodiazepines used in large quantities can also result in a dopamine rush, which is responsible for creating a sense of pleasure and reward [20]. Benzodiazepines are commonly consumed orally but some smoke, snort or inject. They are commonly referred to as "candy", "downers", "sleeping pills", or "tranks" [21]. Flunitrazepam is one of the commonly abused drugs and has been referred to as date rape drug, roofies, or forget-me-pill [22].

Over the past several years there have been rising concerns about the misuse of benzodiazepines. A study has estimated that 30.6 million adults (12.6\%) reported benzodiazepine use in the year 2015-2016 [23]. Among them they report that $2.2 \%$ have misused a BZD prescription. Among adults and adolescents, benzodiazepines are the third most commonly misused illicit or prescription drug in the USA [24,25]. Over the past few decades, benzodiazepine use has increased tremendously. A study done by Bachhuber et al. showed that the number of adults who filled a benzodiazepine prescription increased by $67 \%$, from 8.1 million to 13.5 million between the years 1996 and 2013. The quantity obtained also increased from $1.1 \mathrm{~kg}$ to $3.6 \mathrm{~kg}$ lorazepam-equivalents per 100,000 adults [26].

According to the National Institute of Drug Abuse (NIDA), among 2017-2018 benzodiazepine misusers, $46.3 \%$ of respondents reported that the motivation for their most recent misuse was to relax or relieve tension, followed by helping with sleep in $22.4 \%$. About $5.7 \%$ reported "experimentation" as their main motivation for misuse, and $11.8 \%$ reported using them to "get high" or because of being "hooked". The data also showed that most misusers obtained benzodiazepines from friends or relatives, with only about $20 \%$ receiving them from their doctor [27]. Opioids have been largely involved in deaths related to overdose, and NIDA reports that benzodiazepines have been involved in more than $30 \%$ of opioid-related overdose deaths [27]. Benzodiazepines have also been implicated in causing addiction and long-term cognitive problems. NIDA also reported that among benzodiazepine users, $17.1 \%$ misused them and fewer than $2 \%$ had benzodiazepine use disorder [27]. Benzodiazepine derivatives are prescribed in large quantities globally and are potentially new emerging environmental contaminants. Several studies have emerged showing the presence of BZD derivatives in sewage water and hospital effluents [28-32]. Wastewater samples in Mississippi, USA, have been found to contain alprazolam, $\alpha-\mathrm{OH}$-alprazolam, nordiazepam, oxazepam and temazepam [32]. Scientists in Sweden found that fish living downstream from sewage treatment plants (STP) had elevated benzodiazepine levels and found concentrations of a common benzodiazepine, oxazepam, of $0.73 \mathrm{mg} /$ liter in treated wastewater effluent and $0.58 \mathrm{mg} /$ liter in a midsized stream (River Fyris) receiving input of treated wastewater. These studies indicate that wild fish populations may be affected, and we are still unaware of how these trace levels affect humans [33]. These studies have not only provided information on the prevalence of drug use on a community level, but also warn caution on the use of benzodiazepines.

We hypothesized an increase in benzodiazepine use during the COVID-19 pandemic and evaluated the extent of benzodiazepine use during the COVID-19 pandemic in terms of benzodiazepine prescription and other illicit use.

Table 1 below demonstrates half-life, onset of action and dose equivalency of commonly utilized benzodiazepines. 


\section{Cureus}

\begin{tabular}{|l|l|l|l|}
\hline Benzodiazepine & Half Life in hours & Peak time of action in hrs & Dose equivalent to $5 \mathrm{mg}$ Diazepam \\
\hline Alprazolam & 12 & $1-2$ & $0.5-1$ \\
\hline Chlordiazepoxide & 100 & $1-4$ & $10-25$ \\
Clonazepam & $18-50$ & $1-2$ & $0.25-0.5$ \\
Clorazepate & 100 & $0.5-2$ & $7.5-15$ \\
Diazepam & 100 & $1-2$ & $5-10$ \\
Flurazepam & 100 & $0.5-1$ & $15-30$ \\
\hline Lorazepam & 15 & $1-4$ & $1-2$ \\
\hline Oxazepam & 8 & $1-4$ & $15-30$ \\
Quazepam & $25-41$ & $1-5$ & $10-20$ \\
\hline Temazepam & 11 & $2-3$ & $10-20$ \\
\hline Triazolam & 2 & $1-2$ & $0.25-0.5$ \\
\hline
\end{tabular}

TABLE 1: Table demonstrating half life, onset of action and dose equivalency when compared to $5 \mathrm{mg}$ of diazepam [17,34-38]

\section{Review \\ Methods}

We conducted an extensive literature review using databases PubMed, CINAHL, Cochrane, and Google Scholar (sorted by date and articles with abstracts only were included to review) using the keywords "benzodiazepines" and "COVID-19 Pandemic" following the Preferred Reporting Items for Systematic Review and Meta-Analysis (PRISMA) protocol [39]. Inclusion criteria included research conducted in all countries after the beginning of the coronavirus pandemic, and both English and non-English articles were included. Studies that reported the use of benzodiazepine since the beginning of the pandemic were included. Excluded studies include non-peer-reviewed publications, case reports, case series, review articles, and letters to editors. Studies involving substance abuse but not mentioning specifically benzodiazepine use were also excluded. We included articles from January 1, 2020, to April 30, 2021.

Figure 1 below depicts the process of review and inclusion of articles for the purposes of this review. 


\section{Cureus}

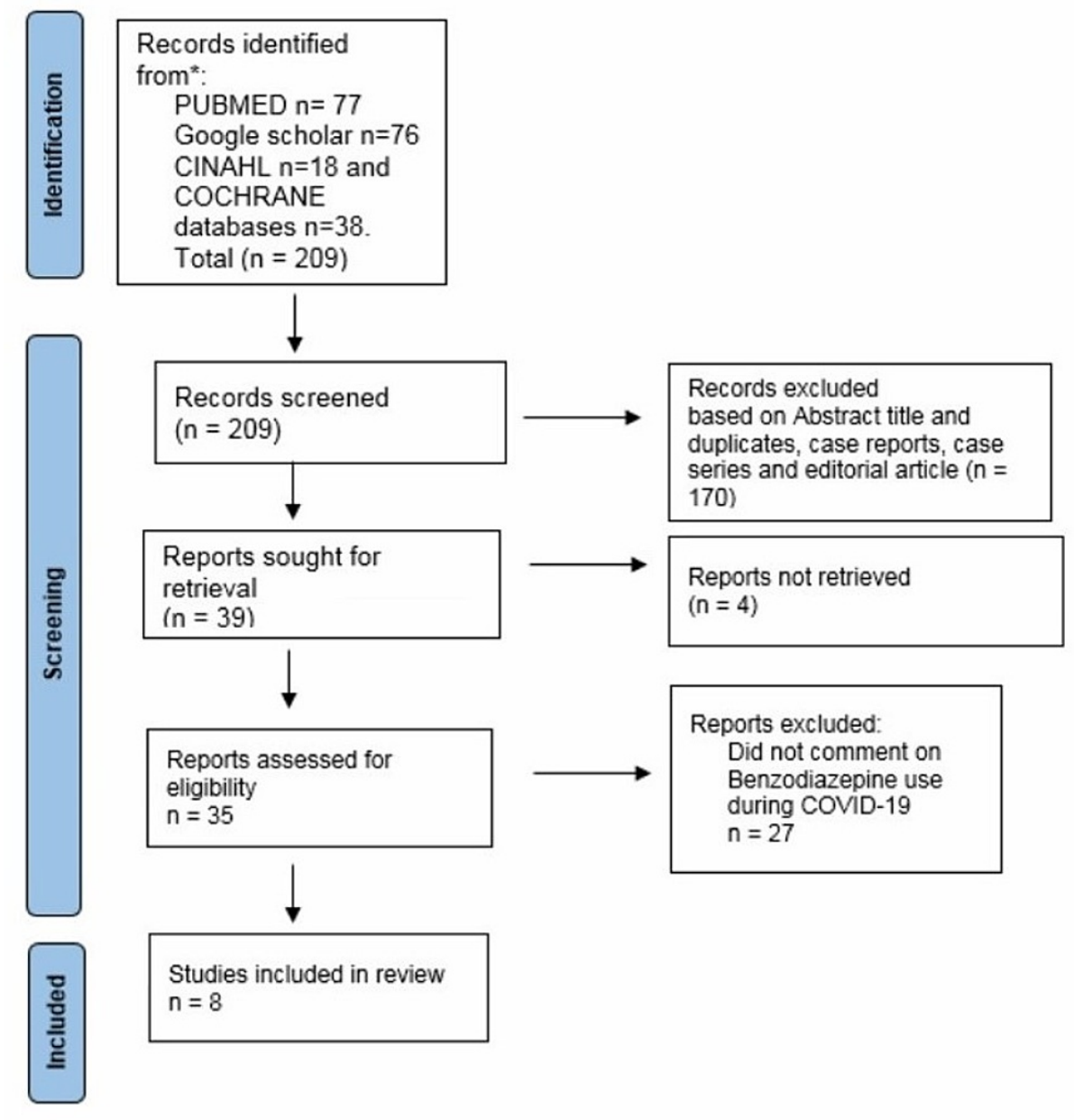

FIGURE 1: Preferred Reporting Items for Systematic Review and MetaAnalysis (PRISMA) flow diagram depicting the process of identification and selection of articles.

\section{Results}

Our search strategy resulted in 209 studies; 31 studies are excluded based on title screening. One hundred seventy articles were excluded for various reasons. Reasons for exclusion included duplicates articles (35), case reports (26), case series (seven), review articles (61), and editorial articles (10). Following this, 39 articles were retrieved out of which four were not accessible. Final full texts of 35 article are reviewed and eight studies written in English which reported the use of benzodiazepines during COVID-19 pandemic are included. The final eight articles were deemed suitable to be included for the conciseness of this review.

\section{Discussion}

Benzodiazepines, especially diazepam, have been the main course of treatment for anxiety and insomnia [40]. Prescribing benzodiazepines has been controversial due to the recognized deleterious effects of longterm treatment with these drugs. Benzodiazepine use in COVID-19 patients has been shown to exacerbate delirium and suppress respiratory drive in patients with respiratory suppression [41]. Caution has been exercised in the use of benzodiazepines, especially palliative care. Some drugs such as midazolam and triazolam are contraindicated with the use of lopinavir/ritonavir because of the risk of increasing the level of some benzodiazepines due to CYP450 inhibition [41]. With the emerging coronavirus pandemic, most countries have updated their drug prescription policies [1]. In the USA, Prescription Drug Monitoring Program (PDMP) is an electronic way to monitor the dispensing of controlled substances used in most states [42].

The COVID-19 pandemic has caused a disruption of the availability of critical mental health services and as a result many people may have faced an increase in the use of alcohol and drugs [1]. Benzodiazepines may have a high potential for abuse and misuse during the pandemic [43], and they are typically co-abused in patients with substance use disorders [44]. The most frequent primary abuse drugs are opioids and/or alcohol; benzodiazepines are misused to enhance the other drug's euphoric effects, reduce the unwanted 
effects of drugs, such as insomnia due to stimulant use, and alleviate withdrawal symptoms between doses [45]. According to a recent study, among 196 participants, 47\% reported an increase in substance use since COVID-19 related to loss of employment, fear of catching the virus, social distancing and isolation. Some people reported substance use as a coping mechanism to deal with the negative physical, mental and social impacts of COVID-19 [46].

In Dublin, measures have been taken promptly after the declaration of the COVID-19 pandemic by the WHO. There was a growing concern about the spread of coronavirus and mortality among people with drug dependency and homelessness. Sixty-two percent of homeless people on opioid substitution therapy also misused street benzodiazepines [47,48]. So, clients were placed in isolation units and shielding units. Patients with benzodiazepine dependency were offered up to $30 \mathrm{mg}$ of benzodiazepine daily to prevent withdrawals for the period of isolation only. In the homeless sector, over 70 people were commenced on benzodiazepine maintenance treatment [49]. In Ireland, it has been reported that $92 \%$ of overdoses where methadone was implicated and $81 \%$ of deaths where heroin was implicated involved benzodiazepines predominantly [47]. In France, because of social isolation or psychological troubles due to lockdown with the potential increase of marital conflicts and domestic violence, there was a prediction for increased consumption of benzodiazepines [50]. Further data collection by the French addictovigilance centers, pharmacies, and addiction specialized centers revealed abuse or misuse with alcohol or other psychoactive substances. Among benzodiazepines, clonazepam, alprazolam, and oxazepam were the most frequently reported to be misused or abused [50].

Lockdown has also resulted in a lot of online browsing about potential drugs of abuse. Google trends in health-related research showed a significant increase in online search interest for the keywords representing benzodiazepines during the lockdown in India [51]. Ireland also reported an increase in the use of alcohol and benzodiazepine use during lockdown periods which has continued after the lockdown as well [52]. The use of illicit drugs has returned to pre-lockdown levels, leading to an elevated risk of developing comorbid psychiatric disorders and other health conditions [52].

The United Nations reports that the non-medical use of benzodiazepines is a well-known phenomenon and represents an increasingly widespread public health problem during the pandemic since its magnitude is difficult to estimate, mainly due to the lack of monitoring and data collection in most countries [53]. This misuse pattern is associated with an elevated risk of serious health consequences or fatal overdose, especially among high-risk opioid users, who misuse benzodiazepines to increase the effects of opioids or to self-medicate to treat symptoms of psychiatric disorders, negative emotional states, opioid withdrawal symptoms, and the side effects of alcohol and cocaine use. The diversion of benzodiazepines most commonly involves alprazolam, diazepam, and lorazepam which seem to have a wider diffusion than oxazepam, clorazepate, and chlordiazepoxide because of their higher abuse potential [53]. In Europe, causes of deaths among high-risk opioid users are more frequently related to the use of benzodiazepines with rapid onset of action such as diazepam, clonazepam, and alprazolam rather than those with a slower onset such as oxazepam and flunitrazepam [54].

In Texas, USA, a study was done to observe the trend in benzodiazepine prescription during the pandemic. According to the Texas Prescription Monitoring Program, the average daily number of patients who filled new benzodiazepine prescriptions was 17,548.56 (Standard Deviation $(S D)=1295.06)$. There was a significant decrease in the benzodiazepine prescriptions between January 5, 2020, and May 12, 2020 ( $\beta=$ $-1982,95 \% \mathrm{CI}=-3712.43,-252.14$ ) [55]. A similar trend was observed with benzodiazepine prescribers [55]. On March 22, 2020, the governor issued an executive order restricting elective medical procedures including many routine outpatient visits. Before the executive order, there was an average of 9,087.24 (SD = 356.26) unique daily prescribers of new benzodiazepine prescriptions. There was a significant decrease in benzodiazepine prescribers associated with the executive order $(\beta=-708.62,95 \% \mathrm{CI}=-1190.54,-226.71)$. This result may be due to a large gap in the care for patients having benzodiazepine prescriptions that has emerged during the pandemic [55]. A study was done by Niles et al. analyzing the deidentified urine specimen samples from the Quest Diagnostics medMATCH ${ }^{\circledR}$ method. There was a significant decrease in non-prescribed benzodiazepine use $(4 \%, \mathrm{p}<0.02)$ indicating a decrease in overall misuse during the pandemic. However, among benzodiazepine users, positivity for non-prescribed fentanyl increased by $48 \%(\mathrm{P}<0.01)[56]$.

In the USA, a study was done comparing the unique dispense of medications from January 2019 to May 2020 [57]. The monthly number of unique patients dispensed as benzodiazepines (mean =4,781,043 [SD = $166,850]$; range $=4,478,448$ to 5,011,279) was relatively stable until March 2020. Since March 2020 the number of unique patients dispensed benzodiazepines $(5,128,721)$ was statistically and significantly higher than forecast estimates. In March 2020, an estimated additional 450,074 (95\% CI:189,999 to 710,149) unique patients were dispensed benzodiazepines, compared to forecast estimates [57]. Another study was done in Italy by analyzing hair samples to see drug use patterns during the COVID-19 pandemic. It revealed the percentage of samples positive for benzodiazepines ranged from $16.7 \%$ (5/30 cases) in the period before the lockdown to $53.3 \%$ ( $16 / 30$ cases, $\mathrm{p}<0.01)$ during the lockdown and remained high $(43.3 \%, 13 / 30$ cases, $\mathrm{p}<0.01)$ even after the lockdown [58]. The benzodiazepines reported in this study were not prescribed and their intake indicated illicit use. The study revealed changes in the overall trend of drug intake during the considered study period; $11 / 30$ (37\%) patients switched from single-drug use in the two pre-lockdown period 
controls to poly-drug use in the post-lockdown period [58]. According to an Italian report during COVID-19, there has been a concerning increase in the prescription of hypnotics/sedatives with the potential for abuse, which has almost doubled and increased by about 17-19\% [59,60]. In another study in Ontario, data from January 1 to May 31, 2019, were compared with data from January 1 to May 31, 2020. There was a 43.7\% increase in benzodiazepine dispensing in the first five months of the year compared to the year prior [61]. The same study also reported that the number of benzodiazepine tablets dispensed monthly during the COVID-19 pandemic was statistically higher compared to the previous year

$(1037.4 \pm 122.24,721.6 \pm 156.87$, respectively, $\mathrm{z}=-2.402, \mathrm{p}=0.016)$ [61].

In Spain, a telephone survey was conducted by the health professionals among the Drug Addiction Assistance of Castile and Leon (DAACYL) units. The professionals in the DAACYL units expressed that the clinical impact during the first six weeks of the pandemic was moderate; however, six centers reported that patients increased or started consuming alcohol and benzodiazepines, especially alprazolam [62]. Among people who were confirmed with coronavirus infection and discharged subsequently, there was a 3.3\% initiation of new benzodiazepine prescriptions [63]. The findings of all the studies discussed are summarized in Table 2.

\begin{tabular}{|c|c|c|c|c|c|}
\hline Author & $\begin{array}{l}\text { Site of } \\
\text { Study }\end{array}$ & Study Period & Data Collection & $\begin{array}{l}\text { Sample } \\
\text { Size }\end{array}$ & Findings \\
\hline $\begin{array}{l}\text { Lapeyre- } \\
\text { Mestre et } \\
\text { al. [50] } 2020\end{array}$ & France & $\begin{array}{l}\text { March } 17^{\text {th }} \\
2020-\text { May } \\
31^{\text {st }}, 2020\end{array}$ & $\begin{array}{l}\text { Data collected from French } \\
\text { Addictovigilance Network }\end{array}$ & $\mathrm{n}=231$ & $\begin{array}{l}\text { Significant misuse and abuse of } \\
\text { benzodiazepines reported (with alcohol or other } \\
\text { psychoactive substances). }\end{array}$ \\
\hline $\begin{array}{l}\text { Downs et } \\
\text { al. [55] } 2020\end{array}$ & USA & $\begin{array}{l}\text { January } 5^{\text {th }} \\
2020 \text {, to May } \\
12^{\text {th }}, 2020\end{array}$ & $\begin{array}{l}\text { Texas Prescription } \\
\text { Monitoring Program. }\end{array}$ & $\mathrm{n}=18000$ & $\begin{array}{l}\text { Significant decrease associated with both } \\
\text { benzodiazepine prescriptions and prescribers. }\end{array}$ \\
\hline $\begin{array}{l}\text { Niles et } \\
\text { al. [56] } 2020\end{array}$ & USA & $\begin{array}{l}\text { January } 1^{\text {st }} \\
2019, \text { through } \\
\text { May } 16^{\text {th }} \\
2020\end{array}$ & $\begin{array}{l}\text { De-identified results from all } \\
\text { medMATCH specimens with } \\
\text { clinician-provided } \\
\text { prescribed drug information }\end{array}$ & $\mathrm{n}=44211$ & $\begin{array}{l}\text { There was a } 4 \% \text { decrease in non-prescribed } \\
\text { benzodiazepine use }(p<0.02)\end{array}$ \\
\hline $\begin{array}{l}\text { Jones et } \\
\text { al. [57] } 2020\end{array}$ & USA & $\begin{array}{l}\text { January } \\
\text { 2019-May } \\
2020\end{array}$ & $\begin{array}{l}\text { Data from the IQVIA Total } \\
\text { Patient Tracker database }\end{array}$ & $n=5,128,721$ & $\begin{array}{l}\text { The results of the study showed additional } \\
450,074(95 \% \text { Cl: } 189,999 \text { to } 710,149) \text { unique } \\
\text { patients were dispensed benzodiazepines } \\
\text { compared to forecasted estimates }\end{array}$ \\
\hline $\begin{array}{l}\text { Gili et } \\
\text { al. [58] } 2020\end{array}$ & Italy & $\begin{array}{l}\text { March } 22^{\text {nd }} \text {, } \\
2020-\text { May } \\
18^{\text {th }}, 2020\end{array}$ & $\begin{array}{l}\text { Hair Sample collected from } \\
30 \text { patients (aged } 18-48 \mathrm{y} ; \\
17 \text { males; } 13 \text { females) from } \\
\text { urban areas of central Italy } \\
\text { for analysis }\end{array}$ & $n=30$ & $\begin{array}{l}\text { The percentage of samples positive for } \\
\text { benzodiazepines increased from } 16.7 \% \text { ( } 5 \\
\text { cases) in the period before the lockdown to } \\
53.3 \% \text { ( } 16 \text { cases, } p<0.01 \text { ) during the lockdown } \\
\text { and remained high after the lockdown }(43.3 \% \text {, } \\
13 \text { cases, } p<0.01 \text { ). }\end{array}$ \\
\hline $\begin{array}{l}\text { Yu et } \\
\text { al. [61] } 2020\end{array}$ & Canada & $\begin{array}{l}\text { January } 1^{\text {st }} \text { to } \\
\text { May } 31^{\text {st }} \\
2019 \text { Vs } \\
\text { January } 1^{\text {st }} \text { to } \\
\text { May } 31^{\text {st }} \text {, } \\
2020\end{array}$ & $\begin{array}{l}\text { Prescription refill } \\
\text { information from } \\
\text { independent community } \\
\text { pharmacy }\end{array}$ & $\mathrm{n}=365$ & $\begin{array}{l}\text { There was significantly more frequent } \\
\text { dispensing of benzodiazepine tablets }(z=2.402 \text {, } \\
p=0.016) \text { in the first five months of } 2020 \\
\text { compared to those of } 2019 \text {. }\end{array}$ \\
\hline $\begin{array}{l}\text { McCarthy } \\
\text { et } \\
\text { al. [63] } 2020\end{array}$ & USA & $\begin{array}{l}\text { March } 7^{\text {th }} \\
2020-\text { March } \\
30^{\text {th }}, 2020\end{array}$ & $\begin{array}{l}\text { Data collected from } \\
\text { Confirmed coronavirus } \\
\text { infection }\end{array}$ & $\mathrm{n}=213$ & $\begin{array}{l}\text { New benzodiazepine initiation was } 3.3 \% \text { among } \\
\text { discharged patients. }\end{array}$ \\
\hline
\end{tabular}

TABLE 2: Summary of significant findings of major studies conducted evaluating benzodiazepine use during the COVID-19 pandemic.

Five out of eight studies showed an increase in benzodiazepine use, whereas two have reported a decrease. These data indicate there have been changing trends in benzodiazepine prescription and misuse during the pandemic. 


\section{Limitations}

We restricted our review to the search terms Benzodiazepines and COVID-19 pandemic, so we may have missed relevant studies which did not have these keywords. We discussed in a broader sense the use of a benzodiazepine during the pandemic including their prescriptions, rather than strictly focusing on benzodiazepine misuse. Several studies which have reported other benzodiazepine use along with opioids and other psychotropics are also included. The studies lacked clear details of benzodiazepine misuse and the individuals might have been using benzodiazepines as prescribed, not representing misuse. We also included studies done using urine and hair sample analysis rather than restricting to strict terms of benzodiazepine misuse.

\section{Conclusions}

We discussed the various trends observed regarding the benzodiazepines use during COVID-19 pandemic as reported in different studies. We reviewed benzodiazepine misuse and predict how the trends of benzodiazepine misuse may vary in the post-pandemic era. COVID-19 has been a global pandemic that has spiked the rates of anxiety and insomnia. Mental health professionals need to be prepared for issues that may arise due to COVID-19 such as anxiety, depression, fear, coronophobia, trauma and grief due to loss of a loved one. The risk of developing benzodiazepine tolerance, abuse and addiction must be considered by health care professionals before prescribing them. This review summarizes the trends observed during the pandemic and emphasizes the importance of weighing risks versus benefits before the prescription of benzodiazepines. The studies which showed an increase in benzodiazepine use represent the tip of an iceberg. We hypothesize the trends in benzodiazepine usage may increase in the future and caution is warranted. We hope mental health professionals prescribe benzodiazepines carefully and make changes in practice according to the prescription drug monitoring program data to avoid a benzodiazepine crisis. Future research is warranted for the periodic assessments of an evolving benzodiazepine crisis.

\section{Additional Information \\ Disclosures}

Conflicts of interest: In compliance with the ICMJE uniform disclosure form, all authors declare the following: Payment/services info: All authors have declared that no financial support was received from any organization for the submitted work. Financial relationships: All authors have declared that they have no financial relationships at present or within the previous three years with any organizations that might have an interest in the submitted work. Other relationships: All authors have declared that there are no other relationships or activities that could appear to have influenced the submitted work.

\section{References}

1. Coronavirus disease (COVID-19) pandemic . (2021). https://www.who.int/emergencies/diseases/novelcoronavirus-2019.

2. The territorial impact of COVID-19: Managing the crisis across levels of government . (2020). Accessed: November 10, 2020: https://www.oecd.org/coronavirus/policy-responses/the-territorial-impact-of-covid19-managing-the-crisis-across-level....

3. Twenge JM, Joiner TE: U.S. Census Bureau-assessed prevalence of anxiety and depressive symptoms in 2019 and during the 2020 COVID-19 pandemic. Depress Anxiety. 2020, 37:954-6. 10.1002/da.23077

4. Vahratian A, Blumberg SJ, Terlizzi EP, Schiller JS: Symptoms of anxiety or depressive disorder and use of mental health care among adults during the COVID-19 pandemic - United States, August 2020-February 2021. MMWR Morb Mortal Wkly Rep. 2021, 70:490-4. 10.15585/mmwr.mm7013e2

5. Bäuerle A, Teufel M, Musche V, et al.: Increased generalized anxiety, depression and distress during the COVID-19 pandemic: a cross-sectional study in Germany. J Public Health (Oxf). 2020, 42:672-8. 10.1093/pubmed/fdaa106

6. Lin LY, Wang J, Ou-Yang XY, et al.: The immediate impact of the 2019 novel coronavirus (COVID-19) outbreak on subjective sleep status. Sleep Med. 2021, 77:348-54. 10.1016/j.sleep.2020.05.018

7. Salari N, Khazaie H, Hosseinian-Far A, et al.: The prevalence of stress, anxiety and depression within frontline healthcare workers caring for COVID-19 patients: a systematic review and meta-regression. Hum Resour Health. 2020, 18:100. 10.1186/s12960-020-00544-1

8. Baenninger A, Silva JA, Hindmarch I, et al.: Good Chemistry: The Life and Legacy of Valium Inventor Leo Sternbach. McGraw-Hill, New York; 2003.

9. Sternbach LH: The discovery of librium. Agents Actions. 1972, 2:193-6. 10.1007/BF01965860

10. Sternbach LH: The benzodiazepine story. J Med Chem. 1979, 22:1-7. 10.1021/jm00187a001

11. Wick JY: The history of benzodiazepines. Consult Pharm. 2013, 28:538-48. 10.4140/TCP.n.2013.538

12. Muñoz FL, Alamo C, Udabe RU, Cuenca E: The historical role of barbiturates in “sleep cures" for psychotic and manic disorders. Psiquiatr Biológica. 2004, 11:242-51.

13. Ashton H: The diagnosis and management of benzodiazepine dependence . Curr Opin Psychiatry. 2005 18:249-55. 10.1097/01.yco.0000165594.60434.84

14. Rickels K: Use of antianxiety agents in anxious outpatients . Psychopharmacology (Berl). 1978, 58:1-17. 10.1007/BF00426784

15. Wesson DR, Smith DE, Ling W, Sabnani S: Substance abuse: Sedative, hypnotic, or anxiolytic use disorders . Psychiatry. Tasman A, Kay J, Lieberman JA (ed): John Wiley \& Sons, Chichester; 2008. 1:1186-1200. 10.1002/9780470515167.ch64 
16. Salzman C: The APA Task Force report on benzodiazepine dependence, toxicity, and abuse . Am J Psychiatry. 1991, 148:151-2. 10.1176/ajp.148.2.151

17. Nielsen S: Benzodiazepines. Curr Top Behav Neurosci. 2017, 34:141-59. 10.1007/7854_2015_425

18. Griffin CE 3rd, Kaye AM, Bueno FR, Kaye AD: Benzodiazepine pharmacology and central nervous systemmediated effects. Ochsner J. 2013, 13:214-223.

19. Ashrafi H, Mobed A, Hasanzadeh M, Babaie P, Ansarin K: Monitoring of five benzodiazepines using a novel polymeric interface prepared by layer by layer strategy. Microchem. J. 2019, 146:121-125. 10.1016/j.microc.2018.12.064

20. Well-Known Mechanism Underlies Benzodiazepines' Addictive Properties . (2012). Accessed: April 19, 2012 : https://archives.drugabuse.gov/news-events/nida-notes/2012/04/well-known-mechanism-underliesbenzodiazepines-addictiv....

21. Benzodiazepines. (2020). Accessed: June 15, 2020: https://www.dea.gov/factsheets/benzodiazepines.

22. What Are Date Rape Drugs and How Do You Avoid Them? . (2015). Accessed: March 16, 2015 : https://archives.drugabuse.gov/blog/post/what-are-date-rape-drugs-and-how-do-you-avoid-them.

23. Maust DT, Lin LA, Blow FC: Benzodiazepine use and misuse among adults in the United States . Psychiatr Serv. 2019, 70:97-106. 10.1176/appi.ps.201800321

24. Center for Behavioral Health Statistics and Quality, 2018b. 2017 National Survey on Drug Use and Health: Detailed Tables. Substance Abuse and Mental Health Services Administration, Rockville, MD. (2018). Accessed: September 7, 2018: https://www.samhsa.gov/data/sites/default/files/cbhsqreports/NSDUHDetailedTabs2017/NSDUHDetailedTabs2017.pdf.

25. Johnston LD, Miech RA, O'Malley PM, Bachman JG, Schulenberg JE, Patrick ME: Monitoring the future national survey results on drug use 1975-2018: overview, key findings on adolescent drug use.. Institute for Social Research (ed): University of Michigan, Ann Arbor; 2019.

26. Bachhuber MA, Hennessy S, Cunningham CO, Starrels JL: Increasing benzodiazepine prescriptions and overdose mortality in the United States, 1996-2013. Am J Public Health. 2016, 106:686-8. 10.2105/AJPH.2016.303061

27. Research suggests benzodiazepine use is high while use disorder rates are low . (2018). Accessed: October 18, 2018: https://www.drugabuse.gov/news-events/science-highlight/research-suggests-benzodiazepine-usehigh-while-use-disorder-....

28. Kosjek T, Perko S, Zupanc M, et al.: Environmental occurrence, fate and transformation of benzodiazepines in water treatment. Water Res. 2012, 46:355-68. 10.1016/j.watres.2011.10.056

29. Stamper B, Gul W, Godfrey M, Gul SW, ElSohly MA: LC-MS-MS method for analysis of benzodiazepines in wastewater during Football Games IV. J Anal Toxicol. 2017, 41:205-13. 10.1093/jat/bkw122

30. Pereira A, Silva L, Laranjeiro C, Lino C, Pena A: Selected pharmaceuticals in different aquatic compartments: Part I-source, fate and occurrence. Molecules. 2020, 25:1026. 10.3390/molecules25051026

31. Cunha DL, Mendes MP, Marques M: Environmental risk assessment of psychoactive drugs in the aquatic environment. Environ Sci Pollut Res Int. 2019, 26:78-90. 10.1007/s11356-018-3556-Z

32. Bishop N, Jones-Lepp T, Margetts M, Sykes J, Alvarez D, Keil DE: Wastewater-based epidemiology pilot study to examine drug use in the Western United States. Sci Total Environ. 2020, 745:140697. 10.1016/j.scitotenv.2020.140697

33. Brodin T, Fick J, Jonsson M, Klaminder J: Dilute concentrations of a psychiatric drug alter behavior of fish from natural populations. Science. 2013, 339:814-5. 10.1126/science.1226850

34. Benzodiazepine Equivalence Table. (2007). Accessed: April 2007: https://www.benzo.org.uk/bzequiv.htm.

35. Agarwal SD, Landon BE: Patterns in outpatient benzodiazepine prescribing in the United States . JAMA Netw Open. 2019, 2:187399. 10.1001/jamanetworkopen.2018.7399

36. Benzodiazepine Equivalence Chart. http://www.mental-health-today.com/rx/benzo.htm.

37. Benzodiazepine Equivalency/Half-Life/Dose Chart. (2003). Accessed: September 19, 2003 http://www.bluelight.org/vb/threads/99918-Benzodiazepine-Equivalency-Half-Life-Dose-Chart.

38. Benzodiazepine Dose Conversions (oral). https://globalrph.com/medcalcs/benzodiazepine-converterdosage-conversions/.

39. Page MJ, McKenzie JE, Bossuyt PM, et al.: The PRISMA 2020 statement: an updated guideline for reporting systematic reviews. BMJ. 2021, 372:n71. 10.1136/bmj.n71

40. Barbui C, Cipriani A, Saxena S: Pharmacological Treatment of Mental Disorders in Primary Health Care . World Health Organization, Geneva; 2009.

41. Bilbul M, Paparone P, Kim AM, et al.: Psychopharmacology of COVID-19. Psychosomatics. 2020, 61:411427. 10.1016/j.psym.2020.05.006

42. D'Souza RS, Lang M, Eldrige JS: Prescription Drug Monitoring Program. StatPearls, Treasure Island; 2021.

43. Careful Prescribing of Benzodiazepines during COVID-19 Pandemic: A Review . (2020). Accessed: October 12, 2020: https://www.mentalhealthjournal.org/articles/careful-prescribing-of-benzodiazepines-duringcovid-19-pandemic-a-review....

44. The TEDS Report: Substance Abuse Treatment Admissions for Abuse of Benzodiazepines . (2011). Accessed: June 2, 2011: https://atforum.com/documents/TEDS028BenzoAdmissions.pdf.

45. Schmitz A: Benzodiazepine use, misuse, and abuse: a review . Ment Health Clin. 2016, 6:120-6. 10.9740/mhc.2016.05.120

46. Ali F, Russell C, Nafeh F, Rehm J, LeBlanc S, Elton-Marshall T: Changes in substance supply and use characteristics among people who use drugs (PWUD) during the COVID-19 global pandemic: a national qualitative assessment in Canada. Int J Drug Policy. 2021, 103237. 10.1016/j.drugpo.2021.103237

47. Drug-related deaths and deaths among drug users in Ireland: 2004-2014. (2017). Accessed: June 7, 2017: https://www.hrb.ie/fileadmin/publications_files/Drugnet61_HRB_site.pdf.

48. Dhalla IA, Mamdani MM, Sivilotti ML, Kopp A, Qureshi O, Juurlink DN: Prescribing of opioid analgesics and related mortality before and after the introduction of long-acting oxycodone. CMAJ. 2009, 181:891-6. 10.1503/cmaj.090784

49. O'Carroll A, Duffin T, Collins J: Harm reduction in the time of COVID- 19: case study of homelessness and drug use in Dublin, Ireland. Int J Drug Pol. 2021, 87: 10.1016/j.drugpo.2020.102966 
50. Lapeyre-Mestre M, Boucher A, Daveluy A, et al.: Addictovigilance contribution during COVID-19 epidemic and lockdown in France. Therapie. 2020, 75:343-54. 10.1016/j.therap.2020.06.006

51. Singh S, Sharma P, Balhara YPS: The impact of nationwide alcohol ban during the COVID-19 lockdown on alcohol use-related internet searches and behaviour in India: an infodemiology study. Drug Alcohol Rev. 2020, 10.1111/dar.13187

52. Columb D, Hussain R, O'Gara C: Addiction psychiatry and COVID-19: impact on patients and service provision. Ir J Psychol Med. 2020, 37:164-8. 10.1017/ipm.2020.47

53. Non-medical use of benzodiazepines: a growing threat to public health? . (2017). Accessed: September 2017: https://www.unodc.org/documents/scientific/Global_SMART_Update_2017_Vol_18.pdf.

54. The misuse of benzodiazepines among high-risk opioid users in Europe . (2018). Accessed: June 7, 2018: https://www.emcdda.europa.eu/topics/pods/benzodiazepines_en.

55. Downs CG, Varisco TJ, Bapat SS, Shen C, Thornton JD: Impact of COVID-19 related policy changes on filling of opioid and benzodiazepine medications. Res Social Adm Pharm. 2021, 17:2005-8. 10.1016/j.sapharm.2020.06.003

56. Niles JK, Gudin J, Radcliff J, Kaufman HW: The opioid epidemic within the COVID-19 pandemic: drug testing in 2020. Popul Health Manag. 2021, 24:S43-51. 10.1089/pop.2020.0230

57. Niles JK, Gudin J, Radcliff J, Kaufman HW: The opioid epidemic within the COVID-19 pandemic: drug testing in 2020. Popul Health Manag. 2021, 24:S43-51. 10.1089/pop.2020.0230

58. Gili A, Bacci M, Aroni K, Nicoletti A, Gambelunghe A, Mercurio I, Gambelunghe C: Changes in drug use patterns during the COVID-19 pandemic in Italy: monitoring a vulnerable group by hair analysis. Int J Environ Res Public Health. 2021, 18:10.3390/ijerph18041967

59. Italian Medicines Agency. Report on drug use during the COVID-19 outbreak . (2020). Accessed: July 29, 2020: https://www.aifa.gov.it/-/uso-dei-farmaci-durante-l-epidemia-covid-19-il-29-luglio-lapresentazione-del-rapporto-aifa..

60. Global Medicine Spending and Usage Trends: Outlook to 2025. . (2021). Accessed: 28 April 2021: https://www.iqvia.com/insights/the-iqvia-institute/reports/global-medicine-spending-and-usage-trendsoutlook-to-2025.

61. Yu C, Boone C, Askarian-Monavvari R, Brown T: Trends in pharmacotherapy for anxiety and depression during COVID- 19: a North York area pilot study. Un Toronto Med J. 2021, 98:41-46.

62. Roncero C, Vicente-Hernández B, Casado-Espada NM, et al.: The impact of COVID-19 pandemic on the Castile and Leon Addiction Treatment Network: a real-word experience. Front Psychiatry. 2020, 11:575755. 10.3389/fpsyt.2020.575755

63. McCarthy CP, Murphy S, Jones-O'Connor M, et al.: Early clinical and sociodemographic experience with patients hospitalized with COVID-19 at a large American healthcare system. EClinicalMedicine. 2020, 26:100504. 10.1016/j.eclinm.2020.100504 